\title{
Vaccine Development for Coronavirus (SARS-CoV- 2) Disease (Covid-19); Lipid Nanoparticles
}

\author{
Ravindra B. Malabadi ${ }^{*}$, Kiran P. Kolkar ${ }^{2}$, Neelambika T. Meti ${ }^{3}$, Raju K. Chalannavar ${ }^{4}$ \\ ${ }^{1,4 *}$ Department of Applied Botany, Mangalore University, Mangalagangotri-574199, Mangalore, Karnataka State, India \\ ${ }^{2}$ Department of Botany, Karnatak Science College, Dharwad, Karnataka state, India \\ ${ }^{3}$ Plant Biotechnology Laboratory, Rajiv Gandhi Institute of IT and Biotechnology, Bharati Vidyapeeth University, Maharashtra \\ State, India \\ Corresponding author* \\ DOI: https://dx.doi.org/10.51244/IJRSI.2021.8312
}

\begin{abstract}
This review aims to highlight the rationale for the development of mRNA-lipid nanoparticle based SARS-CoV-2 vaccines. Severe acute respiratory syndrome coronavirus-2 (SARS-CoV-2) is a novel coronavirus, a major threat to human population and declared as global pandemic viral disease (COVID-19). The detection of double mutation (the mutation sites E484Q and L452R) in a new variant, called B.1.617 in India is very dangerous coronavirus strain is the major concern. India's double mutant strain (B.1.617) could be considered as a variant of concern responsible for the second wave. There are many efforts to develop antiviral drugs or natural remedies or development of vaccine for SARS-CoV-2. Plants were also used as the best expression platforms for the SARS-CoV-2 antigen production. There are many antiviral drugs have been tested against SARS-CoV-2. Nanotechnology has a potentiality in COVID-19 treatment and vaccine development. Nanotechnology based vaccines are safe, easy to design, synthesize, or scale up in larger volume compared to the traditional vaccine approaches. The production of non-replicating mRNA-lipid nanoparticle based vaccines is one of the major breakthrough and promising in the recent development of the production of vaccines. Lipid nanoparticles are biocompatible due to their lipid properties and not harmful to the human body; hence, they can be selectively applied in the fields such as biomedical science particularly in the development of vaccine against SARS-CoV-2.
\end{abstract}

Keywords: Antigen, coronavirus, drugs, mRNA, nanomedicine, lipids, nanoparticles, vaccine

\section{INTRODUCTION}

Cevere acute respiratory syndrome coronavirus 2 (SARS( $\mathrm{CoV}-2$ ) is a novel coronavirus responsible for an ongoing human pandemic (COVID-19) (Wu et al., 2020a, 2020b; Mullard, 2020; McKay et al., 2020; Malabadi et al., 2021a, 2021b; Lombo et al., 2021). During late December of 2019, many people of hospital admitted patients were diagnosed with severe pneumonia of unknown etiology (Wu et al., 2020a, 2020b; Shawky et al., 2020; Zhou et al., 2020a, 2020b). This hospital admitted group was epidemiologically linked to a local seafood market in Wuhan, Hubei Province, People's Republic of China (Shawky et al., 2020; Wu et al., 2020a, 2020b; Shawky et al., 2020). These patients were found to be infected with the novel coronavirus strain, severe acute respiratory syndrome coronavirus 2 (SARSCoV- 2 previously named 2019-nCoV) (Zhou et al., 2020a, 2020b
;Wu et al., 2020a, 2020b; Shawky et al., 2020). Infections with SARS-CoV-2 are now widespread around the globe and are increasing exponentially every day (Wu et al., 2020a, 2020b; Shawky et al., 2020). The transmission of SARS-CoV2 appears to be primarily via aerosols (Zhang et al., 2020). Many countries are affected by the rapid outbreak of SARCoV-2 infections and WHO declared coronavirus disease as global pandemic (Wu et al., 2020a, 2020b; Al-Ahmady and Ali-Boucetta, 2020; Zhang et al., 2020). Non pharmaceutical efforts to control the coronavirus disease (COVID-19) pandemic such as personal preventive actions (eg, handwashing, face covers), environmental cleaning, physical distancing, stay-at-home orders, school and venue closures, and workplace restrictions adopted at the national, state, and local levels have also played significant role. In addition to these public health interventions, development of herd immunity could also provide a defence against COVID-19. The high infection rate, long incubation period, along with mild-to-moderate symptoms make COVID-19 a troubling disease (Shin et al., 2020; Zhou et al., 2020a, 2020b; AlAhmady and Ali-Boucetta, 2020). SARS-CoV-2 is an enveloped ssRNA virus with spike-like glycoproteins protruding from its exterior membrane surface forming a 'corona' (Shin et al., 2020). The four major structural proteins of betacoronaviruses are spike $(S)$ protein, envelope (E) protein, membrane $(\mathrm{M})$ protein, and nucleocapsid $(\mathrm{N})$ protein (Shin et al., 2020). The $\mathrm{S}$ protein is an attractive target for vaccine design because it facilitates viral entry into the host cell during the infection process (Shin et al., 2020; Zhou et al., 2020a, 2020b; Zhang et al., 2020). They are distributed in a wide range of animals and can infect humans, primarily afflicting the pulmonary system and gastrointestinal tract (Shawky et al., 2020; Zhou et al., 2020a, 2020b; Zhang et al., 2020).

Another major problem is the new variant, called B.1.617, was initially detected in India with two mutations, the E484Q and L452R (Double mutant Indian strain). The so-called "double mutation" coronavirus found in India can be considered as a variant of concern (VOC) because of its significant rapid spread and leading to the death of infected people. The B.1.617 strain's mutations are feared to make the variant spread faster and partially evade immunity. This new 
variant is believed to be largely responsible for India's current second wave of the COVID-19 pandemic, with infection rates and hospitalisations on the rise once again. The B.1.617 variant strain has all the hallmarks of a very dangerous virus. Mutations at sites E484 and L452 have been observed separately, but this is the first major viral lineage that combines the two. The double mutation refers to specific changes, among some others, that are denoted by E484Q (glutamate is replaced by glutamine at the 484th spot of the spike protein) and L452R (substitution of leucine with arginine at the 452 nd position). This new variant, which has a so-called double mutation, is thought to be fuelling India's deadlier new wave of cases, and has already begun to overwhelm its hospitals and crematoriums. WHO has concluded that "Having two of these mutations, which have been seen in other variants around the world, are concerning," Further, there was a similarity with mutations that will increase transmission as well as reduce neutralization, possibly stunting the ability of vaccines to curb them. Therefore, both mutations are known to decrease although not completely eliminate the binding of the antibodies created by infection and vaccination. This new strain underscores the insidious nature of viruses and threatens to thwart containment efforts in India, despite measures such as the world's largest lockdown last year. Viruses mutate all the time, as the part of evolutionary biology. Some mutations weaken the virus while others may make it stronger, enabling it to proliferate faster or cause more infections (Malabadi et al., 2021a).

The mRNA to become a promising therapeutic tool in the fields of vaccine development and protein replacement therapy (Shin et al., 2020; Yang, 2021; Pardi et al., 2018; Wang et al., 2020). The mRNA-based vaccines are good candidates for responding to the COVID-19 pandemic (Okay et al., 2020; Alfagih et al., 2021). Nanotechnology based vaccines are easy to design, synthesize, or scale up in a larger volume compared to the traditional vaccine approaches (e.g., inactivated and live-attenuated strains (Malabadi et al., 2021b; Lung et al., 2020; Chauhan et al., 2020; Shin et al., 2020; Vijayan et al., 2019; Mufamadi, 2020; Yang, 2021; Chung et al., 2020; Wang et al., 2020). Nanocarriers to potentiate the success of COVID-19 vaccine in terms of efficacy and safety (Rangayasami et al., 2021; Campos et al., 2020; Chauhan et al., 2020). The mRNA-based COVID-19 vaccine utilizes lipid nanoparticles as a carrier (Pardi et al., 2015, 2018; Jackson et al., 2020; Maruggi et al., 2019; McKay et al., 2020; Chauhan et al., 2020). Therefore, nanodelivery systems are crucial for the successful of in vivo delivery of mRNA to the site of action (Khan et al., 2011; Wang et al., 2020; Alfagih et al., 2021; Guevara et al., 2020; Chauhan et al., 2020). Currently, lipid-based nanodelivery systems are the mostly used for developing mRNA-based vaccines (Alfagih et al., 2021; Chauhan et al., 2020). Polymers and lipid-polymer hybrid nanodelivery systems showed considerable promise in terms of stability, high transfection efficiency, safety profile, and cost effective (Alfagih et al., 2021; Khan et al., 2011;
Yavlovich et al., 2010; Scioli-Montoto et al., 2020; Chauhan et al., 2020;Malabadi et al., 2021b).

Plant production platforms are being used to generate vaccines and antiviral proteins inexpensively at mass scale. Phytodrugs also played an important role as an antiviral compounds and inhibited SARS-Cov-2 activity (Malabadi et al., 2021a; Thota et al., 2020; Chrzanowski et al., 2020; Ahmad et al., 2020; Upadhyay et al., 2020). Many of these natural products have pharmacological or biological activity that can be exploited in pharmaceutical drug discovery and drug design (Malabadi et al., 2021a; Thota et al., 2020; Chrzanowski et al., 2020; Ahmad et al., 2020; Upadhyay et al., 2020; Malabadi, 2008; Malabadi and Vijayakumar, 2008; Malabadi et al., 2009, 2010a, 2010b, 2011a, 2011b; Malabadi et al., 2012a, 2012b, 2012c, 2012d; Malabadi et al., 2016a, 2016c, 2016d; Malabadi et al., 2017a, 2017b; Malabadi et al.. 2018; Malabadi and Chalannavar, 2020)

\section{LIPID NANOPARTICLES}

Liposomes are spherical-enclosed membrane vesicles mainly constructed with a synthetic lipid bi-layer membrane, a cell membranes, to entrap drug inside an aqueous core (Kraft et al., 2014; Yavlovich et al., 2010; Puri et al., 2009). Lipid vesicles or liposomes are composed of phospholipid molecules that form contiguous membrane bi-layers able to entrap solute (Kraft et al., 2014). Liposome and lipid nanoparticle-based therapeutic drugs approved for humans typically contain phosphatidylcholine, as a major membrane building block, with fatty acyl chains of varying lengths and saturation (Kraft et al., 2014; Yavlovich et al., 2010; ScioliMontoto et al., 2020). In some cases, cholesterol is also included to increase rigidity and reduce serum-induced membrane instability because of the serum protein binding (Kraft et al., 2014). Liposomes or lipid vesicles are a pivotal biocompatible and biodegradable drug delivery and formulation platforms (Kraft et al., 2014: Puri et al., 2009). The drug inside the aqueous compartment of the liposomes could be transported to tissue, cell, or intracellular targets. Water-soluble (hydrophilic) agents can be encapsulated in the aqueous core enveloped by the lipid membrane or attached on the membrane surface with lipid conjugated to soluble agents (Kraft et al., 2014). Another significant characteristic of liposome is the potentiality to carry both hydrophobic and hydrophilic compounds (Kraft et al., 2014). Liposome and lipid nanoparticles (constructed with different lipid mixtures) exhibits distinct physical stability, distribution, and circulate for a sufficient amount of time in the body (Kraft et al., 2014). Lipid nanoparticles may have the different percentage of drugs bound to lipid molecules (Kraft et al., 2014; Guevara et al., 2020; Chauhan et al., 2020). Main advantages of lipid nanoparticles is the bi-layer membrane could protect drug from hydrolysis or oxidative degradation, thereby minimizing the toxicity (Kraft et al., 2014; Poovi and Damodhara, 2018; Chauhan et al., 2020). Prolonged drug circulation or residence time in the body may increase the drug bioavailability, and provide sufficient time for drug molecules to arrive at disease 
targets (Kraft et al., 2014; Guevara et al., 2020). Lipidnanoparticles can also act as a potential carrier of drugs, antibodies, proteins, and vaccines (Kraft et al., 2014 Alfagih et al., 2021; Guevara et al., 2020; Chauhan et al., 2020). Now a days, there are many lipid-drug formulations have been approved for the human use (Kraft et al., 2014; Guevara et al., 2020). Very recent example is the mRNA-lipid nanoparticle based vaccine for coronavirus has been clinically approved for human use. Lipid-nanoparticle platforms are safe and effective for the delivery of drugs and vaccines, their use for nucleic acid and gene therapeutics continues to be explored (Kraft et al., 2014; Puri et al., 2009; Guevara et al., 2020; Chauhan et al., 2020).

\section{III. mRNA-LIPID NANOPARTICLE VACCINE}

Liposome or lipid nanoparticle were also used for the presentation of antigen which could potentially stimulate both humoral and cell-mediated immunity (Lung et al., 2020; Kraft et al., 2014; Puri et al., 2009; Poovi and Damodhara, 2018). Further, the method of antigen attachment is essential to the immunogenicity of liposome vaccines (Kraft et al., 2014). Two marketed liposome-based vaccines are Inflexal RV and Epaxal R (Kraft et al., 2014). The former is an influenza vaccine, and the latter is a vaccine against hepatitis A (Kraft et al., 2014). Both are virosomes, which contain functional influenza virus membranes (phospholipids, hemagglutinin, and neuraminidase) and are unilamellar (mono or bi-layer) vesicles approximately $150 \mathrm{~nm}$ in diameter (Kraft et al., 2014). mRNA was chemically modified and encapsulated in liposomes for the successful systemic delivery to tumor sites (Kraft et al., 2014). Successful cytoplasmic delivery of mRNA, encoding for an antigen, resulted in vaccine epitope synthesis of the transfected cells (Kraft et al., 2014). The presence of clearly defined antigens in the cytoplasm can enable presentation of both endogenous and exogenous antigens, and provide T-cell activation while being safe (Reichmuth et al., 2016). In fact, DNA and mRNA vaccines shareed many similarities, where the main difference between the two vaccines is the target location for the delivery of the oligonucleotides (Reichmuth et al., 2016). DNA therapeutics have to reach the nucleus, while for mRNA therapeutics, the cytoplasm is the target (Reichmuth et al., 2016). As a result, mRNA therapeutics are easier to deliver because they do not require crossing the nuclear membrane (Reichmuth et al., 2016). In addition, even if mRNA reaches the nucleus, it does not integrate itself or alters the genome (Reichmuth et al., 2016). Further, cytosolic mRNA has no interaction with the genome (Kraft et al., 2014). Moreover, mRNA essentially represents the minimal genetic information, and is only transiently expressed until the mRNA has been degraded (Reichmuth et al., 2016). The mRNA exhibits self-adjuvating properties that promote cellular immunity (Reichmuth et al., 2016). Finally, mRNA synthesis and purification are fast, easy and low cost when compared with other vaccines (Reichmuth et al., 2016). However, mRNA is highly unstable under physiological conditions (Reichmuth et al., 2016).
Nonviral vectors are becoming the preferred vehicle to deliver mRNA (Reichmuth et al., 2016). Currently, lipid nanoparticles (LNPs) are among the most frequently used vectors for the mRNA delivery (Reichmuth et al., 2016; Chauhan et al., 2020). The most important targets for mRNA vaccines are professional antigen presenting cells (APCs), with dendritic cells (DCs) likely being the most relevant cell type (Reichmuth et al., 2016). The new generation of lipids and lipidoids contains amine groups which maintain a neutral or mildly cationic surface charges at physiological $\mathrm{pH}$, thereby, reducing nonspecific lipid-protein interactions and facilitating oligonucleotide release in the cytosol (Reichmuth et al., 2016).

In general, lipid nanoparticles formed by condensing lipids from an ethanol solution in water (Reichmuth et al., 2016; Poovi and Damodhara, 2018; Chauhan et al., 2020). Depending on the lipid nanoparticles (LNP) synthesis method, mRNA is dissolved in the aqueous phase and encapsulated in the condensation process, or is complexed to the finished lipid nanoparticles (LNPs) in a second step (Reichmuth et al., 2016). The theory of vesicle formation assumes that lipid nanoparticles (LNP) formation is based on the disk-like bilayered fragments whose edges are stabilized by ethanol (Reichmuth et al., 2016). Therefore, rapid mixing of the ethanol-lipid phase with excess water is key for the synthesis of small, uniform lipid nanoparticles (LNPs) (Reichmuth et al., 2016). Newer synthesis methods directly mix the lipidethanol phase with an aqueous solution of mRNA in a small T-piece (Reichmuth et al., 2016). Further, mRNA vaccines can be used for both prophylactic and therapeutic vaccination (Reichmuth et al., 2016). The many advantages over protein or DNA vaccines enable the application of mRNA as a prophylactic against diseases where conventional vaccines have not shown sufficient efficacy (Reichmuth et al., 2016). Lipid nanoparticles (LNPs) protect the mRNA against degradation and assist in endocytosis and endosomal escape (Reichmuth et al., 2016). Adjuvants can be incorporated in LNPs and assist in immune activation and potentially tailoring of the immune response (Reichmuth et al., 2016). Lipid nanoparticles (LNPs) can be targeted to specific cell types by decorating their surfaces with specific ligands (Reichmuth et al., 2016). Lipid nanoparticles (LNP) synthesis, using methods such as microfluidic devices, is robust and upscalable (Reichmuth et al., 2016). Some cationic lipidoids exhibit toxicity and repeated application can induce an immune response against polyethylene glycol (Reichmuth et al., 2016).

\section{FATE OF LIPID-NANOPARTICLES IN ANIMAL STUDIES}

Aliphatic esters in lipid-based nanoparticles (LBNs) can be readily degraded by lipases, especially in the small intestine, where pancreatic lipases are abundant (Qi et al., 2017; Poovi and Damodhara, 2018). Triglycerides, which are common components of lipid-based nanoparticles (LBNs), are first broken down into diglycerides, then to monoglycerides, and finally to fatty acids, in the gastrointestinal tract (GIT) (Qi et 
al., 2017; Poovi and Damodhara, 2018). The in vivo fate of lipid-based nanoparticles (LBNs) is essentially determined by the properties of their lipid compositions (Qi et al., 2017; Poovi and Damodhara, 2018). Lipid-based nanoparticles (LBNs) are rapidly degraded via lipolysis wherever lipases are abundant, especially in the gastrointestinal tract. Further, lipid-based nanoparticles (LBNs) that survived lipolysis can be translocated through the circulation to reach terminal organs or tissues (Qi et al., 2017). Lipid composition, particle size, and surface decoration, as well as the formation of protein corona, are the main factors influencing the in vivo fate of lipid-based nanoparticles (LBNs) (Qi et al., 2017). Lipolysis of lipid-based nanoparticles (LBNs) can also occur in the tissues other than the gastrointestinal tract (GIT), even within cells. Triglycerides in lipid-based nanoparticles (LBNs) can be degraded by lyso-somal acid lipase (LAL) following endocytosis (Qi et al., 2017). An in vitro lipolysis test indicated that the most of lipids can be degraded within 2 hour by lyso-somal acid lipase (LAL) (Qi et al., 2017). In addition, most degraded lipids in cells can be excreted within 24 hours with approximately 50\% of the original level detected in both urine and feces (Qi et al., 2017). It is also certain that lipidbased nanoparticles (LBNs) might not be broken down instantly and can survive for some time, which provides an opportunities for them to be used as nanocarriers for drug delivery and mRNA-lipid nanoparticles for the vaccine development (Qi et al., 2017; Poovi and Damodhara, 2018).

\section{FATE OF mRNA-LIPID NANOPARTICLES-SARS- COV-2 VACCINE}

The spike glycoprotein (S protein) is a key target for vaccine development, therapeutic antibody generation, and the clinical diagnosis of COVID-19 (Wang et al., 2020; Jackson et al., 2020; Shin et al., 2020; Maruggi et al., 2019; McKay et al., 2020). S protein helps the virus to enter target cells, but this endocytosis simultaneously depends on both the binding of S protein to membrane ACE2 receptors and the initiative activation of $\mathrm{S}$ protein by cellular proteases (Wang et al., 2020; Jackson et al., 2020; Shin et al., 2020; Maruggi et al., 2019). Therefore, a vaccine against $S$ protein provides an approach for preventing the proliferation and spread of SARSCoV-2 (Wang et al., 2020; Jackson et al., 2020; Shin et al., 2020; Maruggi et al., 2019). The mRNA-based vaccine targeted to the S protein of SARS-CoV-2 works by active immunization (Lung et al., 2020; Wang et al., 2020; Pardi et al., 2015, 2018). This technique will not use the part of the virus but only recombine mRNA of the $\mathrm{S}$ protein in vitro according to the gene sequence, which is coated with lipid nanoparticles for effective delivery (Wang et al., 2020 Jackson et al., 2020; Shin et al., 2020; Maruggi et al., 2019). Once intramuscular (IM) injected into the muscle, the myocytes take up the lipid nanoparticle (LNPs) and then release the mRNAs into the cytoplasm for translation into the S proteins (Wang et al., 2020). These endogenously synthesized $\mathrm{S}$ proteins will be secreted to activate both humoral and cellular immune responses (Wang et al., 2020).
The mRNA-based vaccines actively induce activation of both B cell responses and $\mathrm{T}$ cell cytotoxicity (Wang et al., 2020; Pardi et al., 2015, 2018). When the target protein is released from the host cell, the antigen-presenting cells will quickly capture and process the heterologous protein (Wang et al., 2020). Then, the presentation of MHC I and MHC II on the surface of the antigen-presenting cell membrane (Wang et al., 2020; Pardi et al., 2015, 2018). This step is important for the subsequent activation of B cells and T cells and is also the key to the humoral and cytotoxic response (Wang et al., 2020; Pardi et al., 2015, 2018; Jackson et al., 2020; Shin et al., 2020; Maruggi et al., 2019).

In general, lipid nanoparticles are composed of phospholipid molecules, PEGylated lipids and cationic or ionizable lipids and some times cholestrol is also used in the production of lipid nanoparticles (Qi et al., 2017). In animal model study, lipid nanoparticles will be circulated in the body for some time and then readily degraded by lipases especially in the small intestine, where pancreatic lipases are abundant (Qi et al., 2017). Triglycerides, which are common components of lipid-based nanoparticles (LBNs), are first broken down into di-glycerides, then to monoglycerides, and finally to fatty acids (Qi et al., 2017). The most degraded lipids in cells can be excreted within $24 \mathrm{~h}$ with approximately $50 \%$ of the original level detected in both urine and feces in animals (Qi et al., 2017). But there are no reports on the fate of lipid nanoparticles when human vaccinated with mRNA-lipid nanoparticles SARS-CoV-2. The possible toxic health effects of these nanoparticles associated with human exposure are unknown. Although at this time, the benefits of nanotechnology dominate our thinking, the potential for undesirable human health outcomes should not be overlooked (Gwinn and Vallyathan, 2006; Korani et al., 2015). In general, lipid nanoparticles are not harmful to human body since liposomes are made up of lipids. The consumption of lipids in our daily diet is very common and helps body system. However, over consumption of bad lipids increases the higher cholesterol level leading to blocking of arteries and ends up in the death of person due to heart attack.

Lipid-based nanoparticles (LNPs) are of $100 \mathrm{~nm}$ size carriers that consist of phospholipids, cholesterol, PEGylated lipids and cationic or ionizable lipids (Igyártó et al., 2020; Hassett et al., 2019). The phospholipids and cholesterol have structural and stabilizing roles, whereas the PEGylated lipids supported prolonged circulation (Igyártó et al., 2020; Kozma et al., 2020; Hassett et al., 2019). However, there is growing number of evidence that polyethylene glycol (PEG) can be immunogenic and repeat the administration of polyethylene glycol (PEG) can induce anaphylactoid, complement activation-related pseudoallergy reaction (Igyártó et al., 2020; Kozma et al., 2020). Humans are likely to develop polyethylene glycol (PEG) antibodies because of exposure to everyday products containing polyethylene glycol (PEG) (Igyártó et al., 2020; Kozma et al., 2020). Therefore, some of the immediate allergic reactions observed with the first shot of 
mRNA-LNP vaccines might be related to pre-existing polyethylene glycol (PEG) antibodies (Igyártó et al., 2020; Kozma et al., 2020). Since these vaccines often required a booster shot, the formation of anti-PEG antibodies is expected after the first shot, and thus, the allergic events are likely to increase upon re-vaccination (Igyártó et al., 2020; Kozma et al., 2020). Polyethylene glycol (PEG) is a synthetic polymer that have been utilized for numerous applications such as osmotic laxatives, food packaging applications, topical medications, cosmetics, and in various other household and consumer products (Thi et al., 2020). Consequently, treating patients who have acquired the anti-PEG antibodies with PEGylated drugs resulted in accelerated blood clearance, allergic events, low drug efficacy, hypersensitivity, and, in some cases, life-threatening side effects (Thi et al., 2020).

Moderna Inc., biotechnology company in collaboration with Vaccine Research Center at the U.S. National Institutes of Health, has developed mRNA vaccines (mRNA-1273) encapsulated in lipid-based nanoparticles (Yang, 2021; Campos et al., 2020). Furthermore, mRNA vaccine (BNT162b2) developed by Pfizer-BioNTech can be considered as a great achievements of nanomedicine (Yang, 2021). For many mRNA-based therapeutics, including BNT162b2 (Pfizer-BioNTech) and mRNA-1273 (Moderna Inc.,), the vehicles of choice are lipid nanoparticles (although other materials have also been used) (Malabadi et al., 2021). Complexed with positively-charged lipids, mRNA is more stable and resistant to RNase-mediated degradation and forms self-assembled virus-sized particles that can be administered via different routes (Nanomedicine and the COVID-19 vaccines, 2020; Pardi et al., 2015, 2018; Jackson et al., 2020; Shin et al., 2020; Maruggi et al., 2019). Once endocytosed, the lipid nanoparticles promote endosomal escape, and release their genetic cargo in the cytosol, where the mRNA is translated into antigenic proteins, kick-starting the immune system machinery into producing neutralizing antibodies (Nanomedicine and the COVID-19 vaccines, 2020) Both BNT162b2 (Pfizer-BioNTech) and mRNA1273 (Moderna Inc.,), deliver mRNA encoding genetic variants of the SARSCoV-2 spike protein that are more stable and immunogenic than the natural protein (Nanomedicine and the COVID-19 vaccines, 2020; Pardi et al., 2015, 2018; Jackson et al., 2020; Shin et al., 2020; Maruggi et al., 2019).

Vaccines based on the cytoplasmic expression of chimeric mRNAs containing with ORF viral sequences possess great potential to translate directly in the cytoplasm and block the chromosomal integration (Wang et al., 2020). Once injected, the delivered mRNA can be processed by immune cells and start to produce targeted protein directly through translation, of which is being followed by activating other immune cells to recognize the newly produced viral protein to make antibodies (Wang et al., 2020; Jackson et al., 2020; Shin et al., 2020; Maruggi et al., 2019).

Glycosylation of viral envelopes is a very common biological phenomenon (Wang et al., 2020). Glycosylation refers to the process in which proteins or lipids are linked to sugar chains by the action of enzymes (Wang et al., 2020). Therefore, the higher the degree of glycosylation, the greater the probability that the virus will escape the immune response and the lower the success rate of vaccine development (Wang et al., 2020). However, SARS-CoV-2 has more than twice the glycan sites of HIV (Wang et al., 2020). This unusual degree of glycosylation means that SARS-CoV-2 may mutate quickly, making the development of a vaccine extremely difficult (Wang et al., 2020). However, the use of mRNA-based vaccine technology has targeted only the $S$ protein, rather than the entire virus particle, may lead to the human immune system producing $\mathrm{S}$ protein antibodies without the influence of viral glycosylation. (Wang et al., 2020; Pardi et al., 2015, 2018; Jackson et al., 2020; Shin et al., 2020; Maruggi et al., 2019).

\section{REFERENCES}

[1] Ahmad A, Rehman MU, Ahmad P, Alkharfy KM (2020) Covid19 and thymoquinone: Connecting the dots. Phytotherapy Research. 34:2786-2789.

[2] Al-Ahmady ZS, Ali-Boucetta H (2020) Nanomedicine and nanotoxicology future could be reshaped post-COVID-19 pandemic. Frontiers in Nanotechnology. 2: 1-11. (doi: 10.3389/fnano.2020.610465).

[3] Alfagih IM, Aldosari B, AlQuadeib B, Almurshedi A, Alfagih MM (2021) Nanoparticles as adjuvants and nanodelivery systems for mRNA-based vaccines. Pharmaceutics. 13(45): 1-27. (https://doi.org/10.3390/pharmaceutics13010045).

[4] Campos EVR, Pereira AES, de Oliveira JL, Carvalho LB, Guilger-Casagrande M, de Lima R Fraceto LF (2020) How can nanotechnology help to combat COVID-19? Opportunities and urgent need. Journal of Nanobiotechnology. 18:125. (https://doi.org/10.1186/s12951-020-00685-4).

[5] Chauhan G, Madou MJ, Kalra S, Chopra V, Ghosh D, MartinezChapa SO (2020) Nanotechnology for COVID-19: Therapeutics and vaccine research. ACS Nano. 14: 7760-7782.

[6] Chrzanowski J, Chrzanowska A, Grabo W (2020) Glycyrrhizin: An old weapon against a novel coronavirus. Phytotherapy Research. 1-8. (https://doi. org/10.1002/ptr.6852).

[7] Chung YH, Beiss V, Fiering SN, Steinmetz NF (2020) COVID-19 Vaccine frontrunners and their nanotechnology design. ACS Nano. (https://dx.doi.org/10.1021/acsnano.0c07197). (www.acsnano.org).

[8] Guevara ML, Persano F, Persano S (2020) Advances in lipid nanoparticles for mRNA-based cancer immunotherapy. Front. Chem. 8: 1-17. 589959. (doi: 10.3389/fchem.2020.589959).

[9] Gwinn MR, Vallyathan V (2006) Nanoparticles: Health effectspros and cons. Environmental Health Perspectives. 114 (12):18181825 .

[10] Hassett KJ, Benenato KE, Jacquinet E, Lee A, Woods A, Yuzhakov O, Himansu S et al., (2019) Optimization of lipid nanoparticles for intramuscular administration of mRNA vaccines. Molecular Therapy: Nucleic Acids. 15: 1-11. (https://doi.org/10.1016/j.omtn.2019.01.013).

[11] Igyártó BZ, Jacobsen S, Ndeupen S (2020) Future considerations for the mRNA-lipid nanoparticle vaccine platform. Preprints. 2020120493. (doi: 10.20944/preprints202012.0493.v2).

[12] Jackson NAC, Kester KE, Casimiro D, Gurunathan S, DeRosa F (2020) The Promise of mRNA vaccines: A Biotech and industrial perspective. Npj Vaccines. 5(1):1-6.

[13] Khan SR, Ganguly A, Malabadi RB, Sunwoo HH, Parashar A, Teixeira da Silva JA, Suresh MR (2011) Targeting strategies and nanocarriers in vaccines and therapeutics. Research in Biotechnology. 2(6):08-20. 
[14] Korani M, Ghazizadeh E, Korani S, Hami Z, MohammadiBardbori A (2015) Effects of silver nanoparticles on human health. Eur. J. Nanomed. 7(1): 51-62.

[15] Kraft JC, Freeling JP, Wang Z, Ho RJY (2014) Emerging research and clinical development trends of liposome and lipid nanoparticle drug delivery systems. J. Pharm Sci. 103(1): 29-52. (doi:10.1002/jps.23773).

[16] Kozma GT, Shimizu T, Ishida T, Szebeni J (2020) Anti-PEG antibodies: Properties, formation, testing and role in adverse immune reactions to PEGylated nano-biopharmaceuticals. Advanced Drug Delivery Reviews. (doi:10.1016/j.addr.2020.07.024).

[17] Lombo L, Singh J, Johnson PA, Johnson JC, Mardon AA (2021). Perceived Isolation and Health: Does isolation and feeling of loneliness pose a risk for severe SARS-CoV-2 infection?. Academia Letters. Article 139. (https://doi.org/10.20935/AL139).

[18] Lung P, Yang J, Li Q (2020) Nanoparticle formulated vaccines: Opportunities and challenges. Nanoscale. 12: 5746-5763.

[19] McKay PF, Hu KA, Blakney AK, Samnuan K, Brown JC (2020) Self- amplifying RNA SARS-CoV-2 lipid nanoparticle vaccine candidate induces high neutralizing antibody titers in mice. Nature Communications. (https://doi.org/10.1038/s41467-020-17409-9).

[20] Malabadi RB (2008) Production of edible vaccines for oral immunization in transgenic plants, current and future prospective. Journal of Phytological Research. 21(1): 1-10.

[21] Malabadi RB, Vijaykumar S (2008) Evaluation of antifungal property of medicinal plants. Journal of Phytological Research. 21(1):139-142.

[22] Malabadi RB, Mulgund GS, Nataraja K (2009) Triacontanol induced somatic embryogenesis and plantlet regeneration in Catharanthus roseus. Journal of Medicinal and Aromatic Plant Sciences. 31: 147-151.

[23] Malabadi RB, Mulgund GS, Nataraja K (2010a) Evaluation of antifungal activity of selected medicinal plants. Journal of Medicinal and Aromatic Plant Sciences. 32(1):42-45.

[24] Malabadi RB, Parashar A, Ganguly A, Suresh MR (2010b) Expression of dengue virus envelope protein in a different plant system. Faculty Research and Development day, Faculty of Pharmacy and Pharmaceutical Sciences, University of Alberta, Edmonton, Canada, 19th November 2010. Abstract No-69, page.

[25] Malabadi RB, Ganguly A, Teixeira da Silva JA, Parashar A, Suresh MR, Sunwoo HH (2011a) Overview of plant-derived vaccine antigens: dengue virus. J. Pharm. Pharm. Sci. 14: 400413.

[26] Malabadi RB, Vijayakumar S, Mulgund GS, Nataraja K (2011b) Induction of somatic embryogenesis in papaya (Carica papaya). Research in Biotechnology. 2(5):40-55.

[27] Malabadi RB, Chalannavar RK, Meti NT, Mulgund GS, Nataraja K, Vijayakumar S (2012a) Synthesis of antimicrobial silver nanoparticles by callus cultures and in vitro derived plants of Catharanthus roseus. Research in Pharmacy. 2(6);18-31.

[28] Malabadi RB, Meti NT, Mulgund GS, Nataraja K, Vijayakumar S (2012b) Synthesis of silver nanoparticles from in vitro derived plants and callus cultures of Costus speciosus (Koen.); Assessment of antibacterial activity. Research in Plant Biology. 2(4):32-42.

[29] Malabadi RB, Mulgund GS, Meti NT, Nataraja K, Vijayakumar S (2012c) Antibacterial activity of silver nanoparticles synthesized by using whole plant extracts of Clitoria ternatea. Research in Pharmacy. 2(4):10-21.

[30] Malabadi RB, Lokare-Naik S, Meti NT, Mulgund GS, Nataraja K, Vijayakumar S (2012d) Synthesis of silver nanoparticles from in vitro derived plants and callus cultures of Clitoria ternatea; Evaluation of antimicrobial activity. Research in Biotechnology. 3(5): $26-38$

[31] Malabadi RB, Chalannavar RK, Sowmyashree K, Supriya S, Nityasree BR, Gleiser RM, Meti NT, Vijayakumar S, Mulgund GS, Gani RS, Nasalapure A, Chougale R, Masti S, Chougale A, Divakar MS, Kasai D, Odhav B, Baijnath H (2016a) Ebola virus: Updates on plant made vaccine development. International Journal of Research and Scientific Innovations. 3(6):4-12.
[32] Malabadi RB, Chalannavar RK, Meti NT, Gani RS, Vijayakumar S, Mulgund GS, Masti S Chougale R, Odhav B, Sowmyashree K, Supriya S, Nityasree BR, Divakar MS (2016c) Insulin plant, Costus speciosus: Ethnobotany and pharmacological updates. Int.J. Curr. Res. Biosci. Plant Biol. 3(7): 151-161.

[33] Malabadi RB, Chalannavar RK, Meti NT, Vijayakumar S, Mulgund GS, Gani RS, Supriya S, Sowmyashree K, Nityasree BR, Chougale A, Divakar MS (2016d) Antidiabetic plant, Gymnema sylvestre R. Br., (Madhunashini): Ethnobotany, phytochemistry and pharmacological updates. International Journal of Current Trends in Pharmacobiology and Medical Sciences. 1(4): 1-17.

[34] Malabadi RB, Chalannavar RK, Supriya S, Nityasree BR, Sowmyashree K, Mulgund GS, Meti NT (2017a) Dengue virus disease: Recent updates on vaccine development. International Journal of Research and Scientific Innovations. 4(7):08-29.

[35] Malabadi RB, Chalannavar RK, Supriya S, Nityasree BR, Sowmyashree K, Mulgund GS, Meti NT (2017b) Dengue virus disease: Current updates on the use of Carica papaya leaf extract as a potential herbal medicine. International Journal of Research and Scientific Innovations. 4(8):36-50.

[36] Malabadi RB, Chalannavar RK, Supriya S, Nityasree BR, Sowmyashree K, Meti NT (2018) Role of botanical drugs in controlling dengue virus disease. International Journal of Research and Scientific Innovations. 5(7): 134-159.

[37] Malabadi RB, Chalannavar RK (2020) Safed musli (Chlorophytum borivilianum): Ethnobotany, phytochemistry and pharmacological updates. Int. J. Curr. Res. Biosci. Plant Biol. 7(11): 25-31.

[38] Malabadi RB, Meti NT, Chalannavar RK (2021a) Role of herbal medicine for controlling coronavirus (SARS-CoV-2) disease (COVID-19). International Journal of Research and Scientific Innovations. 8(2):135-165.

[39] Malabadi RB, Meti NT, Chalannavar RK (2021b) Applications of nanotechnology in vaccine development for coronavirus (SARSCoV-2) disease (Covid-19). International Journal of Research and Scientific Innovations. 8(2): 191-198.

[40] Maruggi G, Zhang C, Li J, Ulmer JB, Yu D (2019) mRNA as a transformative technology for vaccine development to control infectious diseases. Molecular Therapy. 27(4):757-72.

[41] Mufamadi MS (2020) Nanotechnology shows promise for nextgeneration vaccines in the fight against COVID-19. MRS Bulletin. 45.(https://www.cambridge.org/core/terms. https://doi.org/10.1557/).

[42] Mullard A (2020) COVID-19 vaccine development pipeline gears up. The Lancet. 395: 1751-1752.

[43] Nanomedicine and the COVID-19 vaccines (2020) Nature Nanotechnology. 15: 963 (2020). (https://doi.org/10.1038/s41565020-00820-0).

[44] Okay S, Ozcan OO, Karahan M (2020) Nanoparticle-based delivery platforms for mRNA vaccine development. AIMS Biophysics. 7(4): 323-338.

[45] Pardi N, Hogan MJ, Porter FW, Weissman D (2018) mRNA vaccines-A new era in vaccinology. Nature Reviews. Drug Discovery. 17(4):261-279.

[46] Pardi N, Tuyishime S, Muramatsu H, Kariko K, Mui BL, Tam YK, Madden TD, Hope MJ, Weissman D (2015) Expression kinetics of mucleoside-modified mRNA delivered in lipid nanoparticles to mice by various routes. J. Controlled Release. 217: 345-351.

[47] Poovi G, Damodhara N (2018) Lipid nanoparticles: A challenging approach for oral delivery of BCS Class-II drugs. Future Journal of Pharmaceutical Sciences. 4: 191-205.

[48] Puri A, Loomis K, Smith B, Lee JH, Yavlovich A, Heldman E, Blumenthal R (2009) Lipid-based nanoparticles as pharmaceutical drug carriers: From concepts to clinic. Crit Rev Ther Drug Carrier Syst. 26(6): 523-580.

[49] Qi J, Zhuang J, Lu Y, Dong X, Zhao W, Wu W (2017) In vivo fate of lipid-based nanoparticles. Drug discovery. 22 (1): 166-172.

[50] Reichmuth AM, Oberli MA, Jaklenec A, Langer R, Blankschtein D (2016) mRNA vaccine delivery using lipid nanoparticles. Ther. Deliv. 7(5):319-334. 
[51] Scioli-Montoto S, Muraca G, Ruiz ME (2020) Solid lipid nanoparticles for drug delivery: Pharmacological and biopharmaceutical aspects. Front. Mol. Biosci. 7:587997. (doi: 10.3389/fmolb.2020.587997).

[52] Shawky E, Ahmed A, Nada AA, Ibrahim RS (2020) Potential role of medicinal plants and their constituents in the mitigation of SARS-CoV-2: Identifying related therapeutic targets using network pharmacology and molecular docking analyses. The Royal Society of Chemistry. 0: 27961-27983 | 27961. (DOI: 10.1039/d0ra05126h).

[53] Shin MD, Shukla S, Chung YH, Beiss V, Chan SK, Ortega-Rivera OA, Wirth DM, Chen A, Sack M, Pokorski JK, Steinmetz NF (2020) COVID-19 vaccine development and a potential nanomaterial path forward. Nature Nanotechnology. 15:646-655

[54] Thi TTH, Pilkington EH, Nguyen DH, Lee JS, Park KD, Truong NP (2020) The importance of Poly(ethylene glycol) alternatives for overcoming PEG immunogenicity in drug delivery

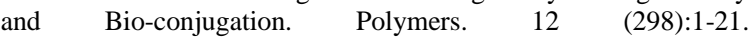
(doi:10.3390/polym12020298).

[55] Thota SM, Balan V, Sivaramakrishnan V (2020) Natural products as home-based prophylactic and symptom management agents in the setting of COVID-19. Phytotherapy Research. 34:3148-3167. (https://doi.org/10.1002/ptr.6794).

[56] Upadhyay S, Tripathi PK, Singh M, Raghavendhar S, Bhardwaj M, Patel AK (2020) Evaluation of medicinal herbs as a potential therapeutic option against SARS-CoV-2 targeting its main protease. Phytotherapy Research. 34:3411-3419.

[57] Vijayan V, Mohapatra A, Uthaman S, Park IK (2019) Recent advances in nanovaccines using biomimetic immunomodulatory materials. Pharmaceutics. 11: 534.
[58] Wang F, Kream RM, Stefano GB (2020) An evidence based perspective on mRNA-SARSCoV-2 vaccine development. Med Sci Monit. 26: e924700; 1-8 (DOI: 10.12659/MSM.924700).

[59] Wu F, Zhao S, Yu B, Chen YM, Wang W, Song ZG, Hu Y, Tao ZW, Tian JH, Pei YY, Yuan ML, Zhang YL, Dai FH, Liu Y, Wang QM, Zheng JJ, Xu L, Holmes EC, Zhang YZ (2020a) A new coronavirus associated with human respiratory disease in China. Nature. 579 (7798) 265-269.

[60] Wu YQ, Zou L, Yu X, Sun D, Li SB, Tang L, Yang JR, Chen $\mathrm{XY}, \mathrm{Wu}$ YG, Fang H (2020b) Clinical effects of integrated traditional Chinese and Western medicine on COVID-19: A systematic review. Shanghai J. Tradit. Chin. Med. 1-8.

[61] Yang (2021) Application of nanotechnology in the COVID-19 pandemic. International Journal of Nanomedicine. 6 :623-649.

[62] Yavlovich A, Smith B, Gupta K, Blumenthal R, Puri A (2010) Light-sensitive lipid-based nanoparticles for drug delivery: Design principles and future considerations for biological applications. Molecular Membrane Biology. 27(7): 364-381. (DOI: 10.3109/09687688.2010.507788).

[63] Zhang R, Lib Y, Zhangc AL, Wangd Y, Molinae MJ (2020) Identifying airborne transmission as the dominant route for the spread of COVID-19. Proc. Natl. Acad. Sci. USA. 117(26): 14857-14863.

[64] Zhou P, Yang XL, Wang XG, Hu B, Zhang L, Zhang W et al., (2020a) Discovery of a novel coronavirus associated with the recent pneumonia outbreak in humans and its potential bat origin. Nature. 579: 270-273. (doi: 10.1038/s41586-020-2012-7).

[65] Zhou P, Yang XL, Wang XG, Hu B, Zhang L, Zhang W et al., (2020b) A pneumonia outbreak associated with a new coronavirus of probable bat origin. Nature. 579: 270-273. 